\title{
ON THE INJECTIVITY AND FLATNESS OF CERTAIN CYCLIC MODULES
}

\author{
V. S. RAMAMURTHI ${ }^{1}$
}

\begin{abstract}
The question of when certain cyclic flat modules of a ring are injective (and vice versa) is studied. The consequences of the conditions 'flat' and 'injective' on the simple modules of a ring are discussed.
\end{abstract}

Introduction. The object of this paper is to consider the relationships between the injectivity and flatness of cyclic modules of the form $R / A$ where $A$ is a two-sided ideal of the ring $R$. In general neither the implication $R / A$ is right injective $\Rightarrow R / A$ is right flat nor its reverse need be true. However, Ware [11] has recently shown that if $R$ is commutative and $A$ is a maximal ideal, then both implications are true. We generalize this observation to noncommutative rings and indicate some conditions under which the injectivity of $R / A$ implies its flatness. These considerations lead to Baer's notion of $p R$-completeness and it turns out that, for simple modules over commutative rings, $p R$-completeness is equivalent to in jectivity, thus yielding a new characterzation of commutative von Neumann regular rings. The paper ends with a discussion of $p R$-completeness and flatness of simple modules over noncommutative rings.

Terminology. The terms ring, module and homomorphism will mean ring with unity, unitary right module and right module homomorphism respectively. Ideal will mean two-sided ideal. If $R$ is a ring and $M$ is a right $R$-module, $M$ will be said to be $I$-complete for a right ideal $I$ of $R$ if any homomorphism $I \rightarrow M$ can be extended to a homomor phism $R \rightarrow M$.

1. Simple modules. Let $A$ be an ideal of $R$. In this section the connection between the injectivity and flatness of the cyclic module $R / A$ is discussed for the case when $A$ is a maximal right ideal.

Received by the editors October 3, 1973 and, in revised form, January 16, 1974. AMS (MOS) subject classifications (1970). Primary 16A30, 16A50, 16A52.

Key words and phrases. Simple module, cyclic module, flatness, injectivity, von Neumann regular ring, $V$-ring.

1 The author wishes to thank the referee for his kind comments. 
(1.1). Lemma. $R / A$ is left $R$-flat if and only if for each $x$ in $A$ there is some $y$ in $A$ such that $x=x y$.

Proof. By Proposition 2.2 of [2], $R / A$ is flat as a left $R$-module if and only if for each $x$ in $A$ there is a left $R$-homomorphism $f: R \rightarrow A$ such that $f(x)=x$. If $y=f(1)$ then $y$ is in $A$ and $x=x y$.

(1.2). Lemma. If $R / A$ is left $R$-flat and $B$ is any right ideal of $R$ such that $B \subset A$ or $B+A=R$ then $R / A$ is $B$-complete.

Proof. Let $f: B \rightarrow R / A$ be a homomorphism. If $B \subset A$ then, by (1.1), for any $x$ in $B$, there is $y$ in $A$ such that $x=x y$ so that $f(x)=f(x) y=0$, as $y$ is in $A$. Thus $f=0$ and hence can be trivially extended to a homomorphism $R \rightarrow R / A$. If $B+A=R$, let $b+a=1$ where $b$ is in $B$ and $a$ is in $A$. Then $x=b x+a x$ for any $x$ in $B$, so that $f(x)=f(b) x+f(a x)$. But, by (1.1), $a x=$ $(a x) t$ for $t$ in $A$. Since $a x$ is in $B$, this gives $f(a x)=0$. Thus $f(x)=f(b) x$ for every $x$ in $B$, which means that $f$ can be extended to a homomorphism $R \rightarrow R / A$.

(1.3). Lemma. Let $A$ be a maximal right ideal of $R$ which is two-sided. If $R / A$ is $p R$-complete for each $p$ in $R$, then $R / A$ is left $R$-flat.

Proof. By (1.1), it suffices to show that for each $a$ in $A, a \in a A$ 。 Consider the epimorphism $f: R / A \rightarrow a R / a A$ defined by $(r+A) \rightarrow(a r+a A)$. If $f=0$ then $a R=a A$ and so $a \in a A$. If $f \neq 0$, then $f$ is an isomorphism and hence $a R / a A$ is $a R-c o m p l e t e$, so that the natural map $a R \rightarrow a R / a A$ is given by left multiplication by an element $(a r+a A)$ of $a R / a A$. This means that $a+a A=(a r+a A) a$, or $(a-a r a) \in a A$. But ara $\in a A$ so that $a \in a A$.

Putting together (1.2) and (1.3) we get

(1.4). Proposition. If $A$ is a maximal right ideal of $R$ which is two-sided, then $R / A$ is left $R$-flat $\Leftrightarrow R / A$ is right $R$-injective $\Leftrightarrow R / A$ is $p R$-complete for each $p$ in $R$ 。

For arbitrary maximal right ideals, this proposition can be reformulated in terms of idealizers as follows. For any right ideal $A$ of $R$, the subring $\bar{A}=\{x \in R ; x A \subset A\}$ is called the idealizer of $A$ in $R . A$ is a two-sided ideal of $\bar{A}$ and $\bar{A} / A \simeq$ End $_{R}(R / A)$ (cf. Robson [9]).

(1.5). Proposition. Let $A$ be a maximal right ideal of $R$. Then $R / A$ is right $R$-flat $\Leftrightarrow \bar{A} / A$ is left $\bar{A}$-injective $\Leftrightarrow \bar{A} / A$ is $\bar{A} p$-complete for every $p$ in $\bar{A}$. 
Proof. Using (1.1), it is easily seen that $R / A$ is right $R$-flat $\Leftrightarrow \bar{A} / A$ is right $\bar{A}$-flat. Now note that $A$ is a maximal left ideal of $\bar{A}$ which is two-sided and apply the left analogue of (1.4).

(1.6). Corollary. If $M$ is a simple module over a commutative ring $R$, then $M$ is flat $\Leftrightarrow M$ is injective $\Leftrightarrow M$ is $p R$-complete for each $p$ in $R$.

(1.7). Corollary. If $R$ is a commutative ring, then $R$ is von Neumann regular if and only if each simple $R$-module is $p R$-complete for each $p$ in $R$.

Remark. The first equivalence in (1.6) was noted by Ware [11] who established it by a different method.

2. Cyclic modules. If $A$ is an arbitrary two-sided ideal of $R$, then neither the implication $R / A$ is right $R$-flat $\Rightarrow R / A$ is right $R$-injective, nor its reverse need be true as shown by the following examples.

Example 1. Let $R=\Pi_{i=1}^{\infty} F_{i}$, where each $F_{i} \simeq Z /(2)$, the field of integers mod 2. Then $R$ is a commutative von Neumann regular ring which is not Artin semisimple and hence has a cyclic flat module which is not injective (cf. Osofsky [6]).

Example 2. Let $K$ be a field, $x$ an indeterminate over $K$ and $W=\left\{\left(a_{i}\right)\right.$; $\left(a_{i}\right)$, a well-ordered sequence of positive real numbers $\}$. Form the ring $T=$ $\left\{\Sigma_{i=0}^{\infty} a_{i} x^{a_{i}} ; a_{i} \in K\right.$ and $\left.\left(\alpha_{i}\right) \in W\right\}$ and consider the factor ring $R=T / x J$ where $J$ is the Jacobson radical of $T . R$ is a commutative local ring with a simple nonzero socle $S=x T / x J$ and $R / S$ is $R$-injective (cf. Caldwell [3]). But $R / S$ cannot be $R$-flat as $R$ is local.

The following proposition gives a condition under which injectivity of $R / A$ implies its flatness.

(2.1). Proposition. Let each principal right ideal of $R$ be projective and $R / A$ be $p R$-complete for each $p$ in $R$. Then $R / A$ is left $R$-flat. $R / A$ will also be right $R$-flat if, further, $R$ contains no nonzero nilpotent elements.

Proof. Let $a \in A$ and $g: a R \rightarrow a R / a A$ be the natural map. Since the homomorphism $f: R / A \rightarrow a R / a A$ defined by $f(r+A)=(a r+a A)$ is an epimorphism and $a R$ is projective, there exists a map $h: a R \rightarrow R / A$ such that $f h=$ $g$. Then, since $R / A$ is aR-complete, $b$ extends to a homomorphism $\bar{b}: R \rightarrow$ $R / A$. Let $\bar{g}=f \bar{h}$. Then $\bar{g}: R \rightarrow a R / a A$ and for any $x$ in $a R, \bar{g}(x)=f(\bar{b}(x))=$ $f(h(x))=g(x)$ and so $\bar{g}$ extends $g$. Hence there is an element $(a r+a A)$ in $a R / a A$ such that $a+a A=(a r+a A) a$, or $(a-a r a) \in a A$, which means that $a \in a A$, since ara $\in a A$. Thus, by (1.1), $R / A$ is left $R-f$ lat. Further, if $a=a x$ 
for some $x$ in $A$, then $(a-x a)^{2}=0$ so that if $R$ has no nonzero nilpotent elements then $a=x a$. Hence, in this case, $R / A$ is also right $R$-flat.

(2.2). Corollary. If $R$ is a commutative ring in which each principal ideal is projective, then any cyclic module which is $p R$-complete for each $p$ in $R$ is flat.

3. More on simple modules. We consider now the validity of (1.7) in the noncommutative case. Call a ring $R$, an $S P I-$ ring, if each simple right module is $p R$-complete for each $p$ in $R$. Rings for which each simple right module is injective have been called $V$-rings and studied by Faith [5] and Cozzens [4]. Since von Neumann regular rings and $V$-rings are $S P I$-rings and there are von Neumann regular rings which are not $V$-rings and vice versa (cf. [4] and [5]), it follows that an SPI-ring need not be a von Neumann regular ring or a $V$-ring. However, SPl-rings have analogous, but weaker, properties.

(3.1). Proposition. Let $R$ be an SPI-ring. Then (i) For each a in $R$, there is an $x$ in RaR such that $a=a x$. (ii) For each ideal $A$ in $R, R / A$ is left $R$-flat. (iii) For each maximal right ideal $M$ of $R$ which is two-sided, $R / M$ is right injective.

Proof. If for some $a$ in $R, a \neq a x$ for any $x$ in $R a R$, then $a \notin a R a R$. Choose a right ideal $K$ maximal with respect to $a R a R \subset K$ and $a \notin K$. Then $X=(a R+K) / K$ is a simple right module and hence $a R$-complete. Thus the natural map $a R \rightarrow X$ is given by left multiplication by an element $(a r+K)$ of $X$. In particular, $a+K=(a r+K) a$, or $(a-a r a) \in K$. Since ara $\in$ aRaR $C$ $K$, this gives $a \in K$, a contradiction. This proves (i). (ii) follows from (i) and (1.1). (iii) follows from (ii) and (1.2).

Rings having the property (i) of (3.1) have been called weakly regular rings and studied in [8]. While, by the above proposition every $S P I-r i n g$ is a weakly regular ring, the converse is not true. For instance, if $R$ is a simple principal right ideal domain which is not a $V$-ring (for an example of such a ring, see [7]), then $R$ is weakly regular but not $S P I$.

Next, call a ring $R$ a right (left) $S F$-ring, if each simple right (left) $R$ module is flat. An $S F$-ring need not be a $V$-ring and vice versa, again by the examples of Faith [5] and Cozzens [4]. While every von Neumann regular ring is a right and left $S F$-ring, we do not know whether the converse is true. However we have

(3.2). Proposition. Let $R$ be a ring such that the left annibilator of any element of $R$ is also a right ideal. If $R$ is a right SF-ring, then $R$ is regular. 
Proof. Let $R$ be right $S F$ and $a \in R$. If $K$ is the left annihilator of $a$ in $R$, then $K$ is also a right ideal by hypothesis. If $M$ is a maximal right ideal of $R$ containing $a R+K$, then, as $R / M$ is flat, $a=x a$ for some $x$ in $M$. Hence $(1-x) \in K \subset M$ or $1 \in M$, a contradiction. Therefore $a R+K=R$ and $a r+k=$ 1 for some $r$ in $R$ and $k$ in $K$. Since $k a=0$, this gives $a=a r a$. Thus $R$ is regular.

We close by noting the following property of $S F$-rings.

(3.3). The centre of any right (left) SF-ring is von Neumann regular.

Proof. From the proof of the above proposition, we have that, if $a \in C$, the centre of $R$, then $a=$ ara for some $r$ in $R$. Now, just as in the proof of the fact that the centre of any regular ring is regular in [10], we can show that $a^{2} r^{3} \in C$ and $a=a\left(a^{2} r^{3}\right) a$.

\section{REFERENCES}

1. R. Baer, Abelian groups that are direct summands of every containing abelian group, Bull. Amer. Math. Soc. 46 (1940), 800-806. MR 2, 126.

2. S. U. Chase, Direct products of modules, Trans. Amer. Math. Soc. 97 (1960), 457-473. MR $22 \# 11017$.

3. W. H. Caldwell, Hypercyclic rings, Pacific J. Math. 24 (1968), 29-44. MR 37 \#1404.

4. J. H. Cozzens, Homological properties of the ring of differential polynomials, Bull. Amer. Math. Soc. 76 (1970), 75-79. MR 41 \#3531.

5. C. Faith, Modules finite over endomorphism ring, Lecture Notes in Math., vol. 246, Springer-Verlag, Berlin, 1972, pp. 146-190.

6. B. L. Osofsky, Rings all of whose finitely generated modules are injective, Pacific J. Math. 14 (1964), 645-650. MR 28 \#5090. $43 \# 6241$.

7. —, On twisted polynomial rings, J. Algebra 18 (1971), 596-607. MR 322.

8. V. S. Ramamurthi, Weakly regular rings, Canad. Math. Bull. 16 (1973), 317-

9. J. C. Robson, Idealizers and hereditary noetherian prime rings, J. Algebra 22 (1972), 45-81. MR 45 \#8687.

10. J. von Neumann, On regular rings, Proc. Nat. Acad. Sci. U. S. A. 22 (1936), 707-713.

11. R. Ware, Endomorphism rings of projective modules, Trans. Amer. Math. Soc. 155 (1971), 233-256. MR 43 \#274.

DE PARTMENT OF MATHEMATICS, ARUL ANANDAR COLLEGE, KARUMATHUR POST, MADURAI DISTRICT, TAMIL NADU, INDIA 